Research Article

\title{
Refinements of Equilibria for Population Games Based on Bounded Rationality of Agents
}

\author{
Chongyi Zhong $\mathbb{D}^{D}$, Hui Yang $(\mathbb{D}$, and Chun Wang \\ Department of Mathematics, Guizhou University, Guiyang, Guizhou 550025, China \\ Correspondence should be addressed to Hui Yang; hui-yang@163.com
}

Received 9 November 2019; Revised 1 March 2020; Accepted 11 March 2020; Published 9 April 2020

Academic Editor: Gen Q. Xu

Copyright (c) 2020 Chongyi Zhong et al. This is an open access article distributed under the Creative Commons Attribution License, which permits unrestricted use, distribution, and reproduction in any medium, provided the original work is properly cited.

We consider stability of equilibria for population games against slight perturbation on the social state space. We provide a necessary and sufficient condition for the existence of Nash equilibria for perturbed population games, which is very important and interesting. Then, refinements of equilibria for population games are introduced. Equivalent characterizations of perfect equilibrium are given. At last, it is shown that each population game admits at least one perfect (proper, weakly proper, and robust) equilibrium.

\section{Introduction and Preliminaries}

Nash [1] proposed the fundamental notion of Nash equilibria for noncooperative games and obtained the Nobel Prize in 1994 due to his contribution to game theory. Much early research in evolutionary game theory has focused on the models generated by a symmetric normal form game. In fact, in Nash's unpublished Ph.D. dissertation [2], he has already provided a "mass action" interpretation for his equilibrium concept, which is close to the perspective of evolutionary game theory. It is a pity that his interpretation seemed to have gone unnoticed for 45 years. Until 1995, many researchers [3-5] reexplored Nash's dissertation and realized the appeal of his interpretation for constructing evolutionary population models. Since then the research of population game theory has proceeded like a raging fire. In fact, early in the 1970s, Maynard and Price [6] introduced the ESS concept as a way of understanding animal conflict. In 1982, Maynard [7] described the interaction of populations consisting of individuals as "playing the field." Weibull [8] provided an overview of the developments that have taken place in evolutionary game theory. The monograph of Sandholm [9] which presents a rigorous and unified description of population game theory was published in 2010.
In a rather long period, many game theorists pay their attention to the definition of rationality of individuals. Consequently, various refinements of equilibria were proposed. See [10-13]. It is worth mentioning that [13] was the first paper of refinements in view of bounded rationality. Actually, early in 1962, Wu and Jiang [14] proposed the notion of essential equilibria for $n$-person noncooperative games and proved that every game can be closely approximated arbitrarily by an essential game.

It is assumed that the agents are always completely rational in [9], which means they are omniscient and omnipotent. This assumption however is too restrictive in reality, especially in population games involving numerous agents. It is reasonable to assume that agents are bounded rational since everyone may make mistakes. Consequently, some agents will sometimes use nonoptimal strategies. Under the bounded rational assumption, we propose some refinements of equilibria for population games, such as perfect equilibria, proper equilibria, weakly proper equilibria, and robust equilibria. Furthermore, we prove the existence of these equilibria and reveal the relations among them.

In this section, we introduce some elementary concepts and notations for population games. In Section 2, perturbed population games are proposed and the concept of perfect 
equilibrium is defined. We proved in Lemma 4 that equilibria for population games with continuous payoff functions are equivalent to solutions of variational inequalities. This conclusion is meaningful and important since the algorithms for solving variational inequalities are abundant. In Section 3, we define proper equilibria and weakly proper equilibria for population games. The existence of that two kinds of equilibria are then proved. In Section 4, robust equilibria for population games are introduced. Although its idea is the same as weakly proper equilibria, our robust equilibria may be better to describe the bounded rationality of agents.

Now some definitions and notations will be given for population games. Let $P=\{1,2, \ldots, p\}$ be a society consisting of $p \geq 1$ populations of agents. The set of strategies for agents in population $p \in P$ is denoted $S^{p}=\left\{1,2, \ldots, n^{p}\right\}$, and $m=\sum_{p \in P} n^{p}$ is the total number of strategies in all populations. $X^{p}=\left\{x^{p} \in R_{+}^{n^{p}}: \sum_{i=1}^{n^{p}} x_{i}^{p}=1\right\}$ denotes the population states for population $p \in P$, where each component $x_{i}^{p}$ of $x^{p}$ represents the share of agents in population $p$ who choose the pure strategy $i$ in $S^{p}$. For each $p \in P$, $C\left(x^{p}\right)=\left\{i \in S^{p}: x_{i}^{p}>0\right\}$ is the support of $x^{p} \in X^{p}$. The society states, denoted $X=\prod_{p \in P} X^{p}$, describe the behavior in all populations.

A payoff function $F: X \longrightarrow R^{m}$ is a continuous map that assigns each social state a vector of payoffs, one for each strategy in each population. For each $p \in P, F_{i}^{p}: X \longrightarrow R$ denotes the payoff function for strategy $i \in S^{p}$, while $F^{p}: X \longrightarrow R^{n^{P}}$ denotes the payoff functions for all strategies in $S^{p}$. A population game is denoted by $G=(P, X, F)$.

The definition of a Nash equilibrium for population games is as follows.

Definition 1 (Definition of [9], p. 24). Let $G=(P, X, F)$ be a population game. A social state $\bar{x} \in X$ is a Nash equilibrium of $G$ if it satisfies:

$$
\text { if } \bar{x}_{i}^{p}>0 \text {, then } F_{i}^{p}(\bar{x})=\max _{k \in S^{p}} F_{k}^{p}(\bar{x}), \quad \forall i \in S^{p}, \forall p \in P .
$$

The notion of best response is now given, and an equivalent characterization of the equilibria for population games will be easily obtained with it.

Definition 2 (Definition of [9], p. 23). Let $G=(P, X, F)$ be a population game. The best response correspondence for population $p \in P$ is given by the following equation:

$$
B^{p}(x)=\left\{y^{p} \in X^{p}: F_{i}^{p}(x)<\max _{k \in S^{p}} F_{k}^{p}(x) \Rightarrow y_{i}^{p}=0\right\}, \quad \forall x \in X .
$$

We say that $y \in X$ is a best response to $x \in X$ if $y^{p}$ is a best response to $x$ for all $p \in P$, that is, $y \in B(x)=\prod_{p \in P} B^{p}(x)$.

Evidently, a social state $x \in X$ is a Nash equilibrium if and only if it is a best response to itself, i.e., $x \in B(x)$. Therefore, we can prove the existence of Nash equilibria for population games by Kakutani fixed point theorem.
Lemma 1 (Theorem of [9], p. 24). Every population game possesses at least one Nash equilibrium.

An important conclusion we will use is stated as follows.

Lemma 2 (Corollary of [15], p. 148). Let $X \subset R^{m}$ be nonempty, compact, and convex, and let $F: X \longrightarrow R^{m}$ be continuous. Then, there exists $\bar{x} \in X$ such that the following variational inequality holds:

$$
\langle\bar{x}-y, F(\bar{x})\rangle \geq 0, \quad \forall y \in X .
$$

Lemma 3 (Kakutani fixed point theorem, Theorem 13 of [16], p. 344). Let $X$ be a compact convex subset and $\Phi$ an upper semicontinuous map from $X$ to $X$ with compact convex images. Then. there exists a fixed point $\bar{x} \in X$ of $\Phi$.

\section{Perfect Equilibria}

As stated in Introduction, we assume that agents are bounded rational; thus, a small number of agents may make mistakes and use nonoptimal strategies. Mathematically, this idea is modelled via perturbed population games, in which every strategy in each population may be chosen by agents.

Definition 3. Let $G=(P, X, F)$ be a population game, and for each population, $p \in P$ and $\eta^{p}=\left(\eta_{1}^{p}, \eta_{2}^{p}, \ldots, \eta_{n^{p}}^{p}\right)$ with $\eta_{i}^{p}>0$ for all $i \in S^{p}$ and $\sum_{i=1}^{n^{p}} \eta_{i}^{p}<1$ be given a perturbation parameter vector. The perturbed population state set of population $p \in P$ is given by the following equation:

$$
X^{p}\left(\eta^{p}\right)=\left\{x^{p} \in X^{p}: x_{i}^{p} \geq \eta_{i}^{p}, \forall i \in S^{p}\right\} .
$$

Furthermore, let $\eta=\left(\eta^{1}, \eta^{2}, \ldots, \eta^{p}\right)$ and $X(\eta)=$ $\prod_{p \in P} X^{p}\left(\eta^{p}\right)$. Then, $G(\eta)=(P, X(\eta), F)$ is the perturbed population game for the population game $G$ with perturbation $\eta$.

The Nash equilibrium concept for perturbed population games is analogous to Definition 1.

Definition 4. Let $G(\eta)=(P, X(\eta), F)$ be a perturbed population game. A social state $\bar{x} \in X(\eta)$ is a Nash equilibrium of $G(\eta)$ if it satisfies:

$$
\text { if } \bar{x}_{i}^{p}>\eta_{i}^{p} \text {, then } F_{i}^{p}(\bar{x})=\max _{k \in S^{p}} F_{k}^{p}(\bar{x}), \quad \forall i \in S^{p}, \forall p \in P \text {. }
$$

Notice that the social states set for a perturbed population game $X(\eta)$ is a subset of $X$ of the original population game, so one may query the existence of an equilibrium for perturbed population games.

Lemma 4. Let $G(\eta)=(P, X(\eta), F)$ be a perturbed population game. Then, $\bar{x} \in X(\eta)$ satisfies

$$
\langle\bar{x}-y, F(\bar{x})\rangle \geq 0, \quad \forall y \in X(\eta),
$$

if and only if $\bar{x}$ is a Nash equilibrium of $G(\eta)$.

Proof. Suppose $\bar{x} \in X(\eta)$ satisfies (6). If $\bar{x}$ is not a Nash equilibrium of $G(\eta)$, then 


$$
\exists p_{0} \in P, \exists i_{0} \in S^{p_{0}}: F_{i_{0}}^{p_{0}}(\bar{x})<\max _{k \in S^{P_{0}}} F_{k}^{p_{0}}(\bar{x}) \text { and } \bar{x}_{i_{0}}^{p_{0}}>\eta_{i_{0}}^{p_{0}} .
$$

Let the pure strategy $j \in S^{p_{0}}$ be such that $F_{j}^{p_{0}}(\bar{x})=\max _{k \in S^{p_{0}}} F_{k}^{p_{0}}(\bar{x}) \quad$ and $\quad y=\left(\bar{x}_{1}^{1}, \ldots, \eta_{i_{0}}^{p_{0}}, \ldots\right.$, $\left.\bar{x}_{j}^{p_{0}}+\bar{x}_{i_{0}}^{p_{0}}-\eta_{i_{0}}^{p_{0}}, \ldots, \bar{x}_{n^{p}}^{p}\right)$, in which each component is the same as the corresponding component in $\bar{x}$ except the $i_{0}^{\text {th }}$ and the $j^{\text {th }}$ components of population $p_{0}$. Obviously, $y \in X(\eta)$. Then, we have

$$
\langle\bar{x}-y, F(\bar{x})\rangle=\left(\bar{x}_{i_{0}}^{p_{0}}-\eta_{i_{0}}^{p_{0}}\right)\left(F_{i_{0}}^{p_{0}}(\bar{x})-F_{j}^{p_{0}}(\bar{x})\right)<0 .
$$

This contradicts with (6).

Conversely, suppose $\bar{x}$ is a Nash equilibrium of $G(\eta)$. Take $\alpha^{p}=\max _{k \in S^{p}} F_{k}^{p}(\bar{x})$ for each $p \in P$. Since $\bar{x}$ is a Nash equilibrium of $G(\eta)$, we have

$$
\text { if } \bar{x}_{i}^{p}>\eta_{i}^{p} \text {, then } F_{i}^{p}(\bar{x})=\alpha^{p}, \quad \forall i \in S^{p}, \forall p \in P .
$$

It is obvious that

$$
\text { if } \bar{x}_{i}^{p}=\eta_{i}^{p} \text {, then } F_{i}^{p}(\bar{x}) \leq \alpha^{p}, \quad \forall i \in S^{p}, \forall p \in P .
$$

For each $p \in P$ and $i \in S^{p}$, we set $\lambda_{i}^{p}=\alpha^{p}-F_{i}^{p}(\bar{x})$, then we have

$$
\lambda_{i}^{p} \begin{cases}=0, & \text { if } \bar{x}_{i}^{p}>\eta_{i}^{p}, \\ \geq 0, & \text { if } \bar{x}_{i}^{p}=\eta_{i}^{p} .\end{cases}
$$

Hence, for each $p \in P, \quad F^{p}(\bar{x})=\left(\alpha^{p}, \ldots, \alpha^{p}\right)^{T}$ $-\sum_{i \in S^{p}} \lambda_{i}^{p} e^{i}$, where $e^{i}$ is the $i^{\text {th }}$ unit vector in $R^{n^{p}}$ for all $i \in S^{p}$. Then, for each $p \in P$, since $\sum_{i \in S^{p}} \bar{x}_{i}^{p}=1$,

$$
\left\langle\bar{x}^{p}, F^{p}(\bar{x})\right\rangle=\alpha^{p}-\sum_{i \in S^{p}} \lambda_{i}^{p} \eta_{i}^{p} .
$$

For each $p \in P$ and $y^{p} \in X^{p}\left(\eta^{p}\right)$, since $\sum_{i \in S^{P}} y_{i}^{p}=1$ and $y_{i}^{p} \geq \eta_{i}^{p}$ for all $i \in S^{p}$, we have

$$
\left\langle y^{p}, F^{p}(\bar{x})\right\rangle=\alpha^{p}-\sum_{i \in S^{p}} \lambda_{i}^{p} y_{i}^{p} \leq \alpha^{p}-\sum_{i \in S^{p}} \lambda_{i}^{p} \eta_{i}^{p}=\left\langle\bar{x}^{p}, F^{p}(\bar{x})\right\rangle .
$$

Therefore, for each $p \in P$ and $y^{p} \in X^{p}\left(\eta^{p}\right)$,

$$
\left\langle\bar{x}^{p}-y^{p}, F^{p}(\bar{x})\right\rangle \geq 0 .
$$

Then, we have

$$
\langle\bar{x}-y, F(\bar{x})\rangle \geq 0, \quad \forall y \in X(\eta) .
$$

The proof is complete.

Lemma 4 implies that the solution of the variational inequality (6) which is defined on the nonempty, compact, and convex set $X(\eta)$ must be a Nash equilibrium of the perturbed population game $G(\eta)$. Moreover, the variational inequality (6) possesses at least one solution on the perturbed social state space $X(\eta)$ according to Lemma 2. Then, we have the following theorem.

Theorem 1. Every perturbed population game admits at least one Nash equilibrium.
With the concepts having been given above, we now give the formal definition of a perfect equilibrium.

Definition 5. Let $G=(P, X, F)$ be a population game. A social state $\bar{x} \in X$ is a perfect equilibrium of $G$ if there exist some sequences $\left\{\eta^{(n)}\right\}_{n=1}^{\infty}$ and $\left\{\bar{x}^{(n)}\right\}_{n=1}^{\infty}$ satisfying the following:

(1) $\lim _{n \rightarrow \infty} \eta^{(n)}=0$

(2) $\vec{n}^{(n)}$ is a Nash equilibrium of the perturbed population game $G\left(\eta^{(n)}\right)$ for each $n$

(3) $\lim _{n \rightarrow \infty} \bar{x}^{(n)}=\bar{x}$

Lemma 5. Let $G=(P, X, F)$ be a population game. Then, every perfect equilibrium of $G$ is a Nash equilibrium of $G$.

Proof. Suppose that the perfect equilibrium $\bar{x}$ of $G$ is not a Nash equilibrium of $G$. Then,

$$
\exists p_{0} \in P, \exists i_{0} \in S^{p_{0}}: F_{i_{0}}^{p_{0}}(\bar{x})<\max _{k \in S^{P_{0}}} F_{k}^{p_{0}}(\bar{x}) \text { and } \bar{x}_{i_{0}}^{p_{0}}>0 .
$$

Since $\bar{x}$ is a perfect equilibrium, there exist sequences $\left\{\eta^{(n)}\right\}_{n=1}^{\infty}$ and $\left\{\bar{x}^{(n)}\right\}_{n=1}^{\infty}$ satisfying $\lim _{n \longrightarrow \infty} \eta^{(n)}=0, \lim _{n \longrightarrow \infty}$ $\bar{x}^{(n)}=\bar{x}$, and $\bar{x}^{(n)}$ is a Nash equilibrium of the perturbed population game $G\left(\eta^{(n)}\right)$ for each $n$. In view of the continuity of $F$ and the continuity of the maximum operator, it is clear that

$$
\begin{aligned}
F_{i_{0}}^{p_{0}}\left(\bar{x}^{(n)}\right) & \longrightarrow F_{i_{0}}^{p_{0}}(\bar{x}), \\
\max _{k \in S^{p_{0}}} F_{k}^{p_{0}}\left(\bar{x}^{(n)}\right) & \longrightarrow \max _{k \in S^{p_{0}}} F_{k}^{p_{0}}(\bar{x}),
\end{aligned}
$$

as $n \longrightarrow \infty$.

Consequently, for sufficiently large $n$, according to (16) and (17) and the property of limits, we have

$$
F_{i_{0}}^{p_{0}}\left(\bar{x}^{(n)}\right)<\max _{k \in S^{p_{0}}} F_{k}^{p_{0}}\left(\bar{x}^{(n)}\right) .
$$

Because $\bar{x}^{(n)}$ is a Nash equilibrium of the perturbed population game $G\left(\eta^{(n)}\right)$, we have that, for sufficiently large $n, \bar{x}_{i}^{(n) p_{0}}=\eta_{i_{0}}^{(n) p_{0}}$. Since $\bar{x}_{i_{0}}^{(n) p_{0}} \longrightarrow \bar{x}_{i_{0}}^{p_{0}}$ and $\eta_{i_{0}}^{(n) p_{0}} \longrightarrow 0$ as $n \stackrel{i}{\longrightarrow} \infty$, we have $\bar{x}_{i_{0}}^{p_{0}}=0$.

This contradicts with our assumption $\bar{x}_{i_{0}}^{p_{0}}>0$. Hence, a perfect equilibrium of $G$ must be a Nash equilibrium of $G$. The proof is complete.

Since the perfectness concept only requires Nash equilibrium to be robust with respect to some low-share perturbation, existence is easy to establish.

Theorem 2. Every population game G possesses at least one perfect equilibrium.

Proof. For any perturbed population game sequence $\left\{G\left(\eta^{(n)}\right)\right\}_{n=1}^{\infty}$ for the population game $G$ with $\eta^{(n)} \longrightarrow 0$ as $n \longrightarrow \infty$, according to Theorem $1, G\left(\eta^{(n)}\right)$ possesses at least one Nash equilibrium $\bar{x}^{(n)}$ for each $n$.

Since $\left\{\bar{x}^{(n)}\right\}_{n=1}^{\infty}$ is a sequence in the compact set $X$, we can assume without loss of generality that $\left\{\bar{x}^{(n)}\right\}_{n=1}^{\infty}$ converges to 
some $\bar{x} \in X$. Then, $\bar{x}$ is a perfect equilibrium of $G$. The proof is complete.

Next, we will give another description for perfect equilibria which is similar to Myerson's definition in [11].

Definition 6. Let $G=(P, X, F)$ be a population game. A social state $x \in X$ is completely mixed if each component of $x$ is positive, that is, $x_{i}^{p}>0$, for each $i \in S^{p}$ and $p \in P$.

Definition 7. Let $G=(P, X, F)$ be a population game and let $\epsilon>0$. A completely mixed social state $\bar{x} \in X$ is an $\epsilon$-perfect equilibrium of $G$ if it satisfies:

$$
\text { if } F_{i}^{p}(\bar{x})<\max _{k \in S^{p}} F_{k}^{p}(\bar{x}) \text {, then } \bar{x}_{i}^{p} \leq \epsilon, \quad \forall i \in S^{p}, \forall p \in P .
$$

Next theorem shows that the above characterization of perfect equilibria is equivalent.

Theorem 3. Let $G=(P, X, F)$ be a population game. The following assertions are equivalent:

(1) $\bar{x} \in X$ is a perfect equilibrium of $G$

(2) There exist some sequences $\left\{\epsilon_{n}\right\}_{n=1}^{\infty}$ and $\left\{\bar{x}\left(\epsilon_{n}\right)\right\}_{n=1}^{\infty}$ such that $\bar{x}\left(\epsilon_{n}\right) \longrightarrow \bar{x}$ and $\epsilon_{n} \longrightarrow 0$ as $n \longrightarrow \infty$, where $x\left(\epsilon_{n}\right)$ is an $\epsilon_{n}$-perfect equilibrium of $G$ for all $n$

(3) There exists a sequence $\left\{\epsilon_{n}\right\}_{n=1}^{\infty}$ and a completely mixed social state sequence $\left\{\bar{x}\left(\epsilon_{n}\right)\right\}_{n=1}^{\infty}$, such that $\bar{x}\left(\epsilon_{n}\right) \longrightarrow \bar{x}$ and $\epsilon_{n} \longrightarrow 0$ as $n \longrightarrow \infty$, with the property that $\bar{x}$ is a best response to $\bar{x}\left(\epsilon_{n}\right)$ for all $n$.

Proof $(1) \Rightarrow(2)$ : since $\bar{x}$ is a perfect equilibrium of $G$, there exists a sequence $\left\{\eta^{(n)}\right\}_{n=1}^{\infty}$ such that $\lim _{n \longrightarrow \infty} \eta^{(n)}=0$ and $\lim _{n \longrightarrow \infty} \bar{x}^{(n)}=\bar{x}$ with $\bar{x}^{(n)}$ a Nash equilibrium of the perturbed population game $G\left(\eta^{(n)}\right)$ for all $n$. Then, we have

$$
\text { if } F_{i}^{p}\left(\bar{x}^{(n)}\right)<\max _{k \in S^{p}} F_{k}^{p}\left(\bar{x}^{(n)}\right) \text {, then } \bar{x}_{i}^{(n) p}=\eta_{i}^{(n) p}, \quad \forall i \in S^{p}, \forall p \in P \text {. }
$$

Let $\epsilon_{n}=\max _{p, i} \eta_{i}^{(n) p}$, then $\bar{x}^{(n)}$ is an $\epsilon_{n}$-perfect equilibrium of $G$ for all $n$.

$(2) \Rightarrow(3)$ : we show that if $\bar{x}_{i}^{p}>0$, then $F_{i}^{p}\left(\bar{x}\left(\epsilon_{n}\right)\right)=\max _{k \in S^{p}} F_{k}^{p}\left(\bar{x}\left(\epsilon_{n}\right)\right)$ for sufficiently large $n$. Since $\lim _{n \longrightarrow \infty} \bar{x}\left(\epsilon_{n}\right)=\bar{x}$ and $\lim _{n \longrightarrow \infty} \epsilon_{n}=0$, we have $\bar{x}_{i}^{p}\left(\epsilon_{n}\right)>\epsilon_{n}$ for sufficiently large $n$. Since $\bar{x}\left(\epsilon_{n}\right)$ is an $\epsilon_{n}$-perfect equilibrium of $G$ for each $n$, this implies $F_{i}^{p}\left(\bar{x}\left(\epsilon_{n}\right)\right)=$ $\max _{k \in S^{p}} F_{k}^{p}\left(\bar{x}\left(\epsilon_{n}\right)\right)$ for sufficiently large $n$.

$(3) \Rightarrow(1)$ : since $\lim _{n \rightarrow \infty} \epsilon_{n}=0$, without loss of generality for every $p \in P$, there exists $j \in S^{p}$ such that $\bar{x}_{j}^{p}\left(\epsilon_{n}\right)>\epsilon_{n}$ for all $n$. For each $p \in P$, define $\eta^{(n) p}$, $n=1,2, \ldots$, as

$$
\eta_{i}^{(n) p}= \begin{cases}\bar{x}_{i}^{p}\left(\epsilon_{n}\right) & i \notin C\left(\bar{x}^{p}\right) \\ \epsilon_{n} & i \in C\left(\bar{x}^{p}\right)\end{cases}
$$

Obviously, for all $n$ and $p \in P, \eta^{(n) p}$ satisfies $\eta_{i}^{(n) p}>0$ for all $i \in S^{p}$ and $\sum_{i \in S^{p}} \eta_{i}^{(n) p}<1$.

As $\epsilon_{n}$ converges to 0 as $n \longrightarrow \infty$, those $\eta_{i}^{(n) p}$ which are equal to $\epsilon_{n}$ converge to 0 . Since $\bar{x}\left(\epsilon_{n}\right)$ converges to $\bar{x}$ as $n \longrightarrow \infty$, for $p \in P$ and $i \notin C\left(\bar{x}^{p}\right)$, we have $\lim _{n \longrightarrow \infty} \bar{x}_{i}^{p}\left(\epsilon_{n}\right)=0$. Therefore, $\eta^{(n)}$ converges to 0 as $n \longrightarrow \infty$. Then, we obtain a sequence of perturbed population games $\left\{G\left(\eta^{(n)}\right)\right\}_{n=1}^{\infty}$.

In the following, it will be shown that $\bar{x}\left(\epsilon_{n}\right)$ is a Nash equilibrium of the perturbed population game $G\left(\eta^{(n)}\right)$ for all $n$. For $p \in P$ and $i \in S^{p}$, if $F_{i}^{p}\left(\bar{x}\left(\epsilon_{n}\right)\right)<\max _{k \in S^{p}} F_{k}^{p}\left(\bar{x}\left(\epsilon_{n}\right)\right)$, then $\bar{x}_{i}^{p}=0$, since $\bar{x}$ is a best response to $\bar{x}\left(\epsilon_{n}\right)$ for all $n$. Then, $i \notin C\left(\bar{x}^{p}\right)$, and according to the definition of $\eta_{i}^{(n) p}$, we have $\bar{x}_{i}^{p}\left(\epsilon_{n}\right)=\eta_{i}^{(n) p}$. Therefore, $\bar{x}\left(\epsilon_{n}\right)$ is a Nash equilibrium of the perturbed population game $G\left(\eta^{(n)}\right)$ for all $n$. The proof is complete.

We conclude this section with the following example, which shows that the concept of perfectness really refines the Nash equilibria for population games.

Example 1. Let $G$ be a population game consisting of two populations, and each population has two strategies. Therefore, the social state space is $X=X^{1} \times X^{2}$, where $X^{i}=\left\{\left(x_{1}^{i}, x_{2}^{i}\right) \in R_{+}^{2}: x_{1}^{i}+x_{2}^{i}=1\right\}, i=1,2$. Payoffs of $G$ are defined, respectively, by $F^{1}(x)=\left(x_{1}^{2}, x_{1}^{2}+3 x_{2}^{2}\right)^{T}$ and $F^{2}(x)=\left(2 x_{1}^{1}+x_{2}^{1}, 2 x_{1}^{1}+4 x_{2}^{1}\right)^{T}$ for any $x \in X$. It is easy to be verified that the two pure social states $((1,0),(1,0))$ and $((1,0),(1,0))$ are both Nash equilibria of $G$. We show that the only perfect equilibrium is $((1,0),(1,0))$. For any $\epsilon \epsilon(0,1)$, let $x(\epsilon)=((\epsilon, 1-\epsilon),(\epsilon, 1-\epsilon))$, It is clear from Definition 7 that each $x(\epsilon)$ is an $\epsilon$-perfect equilibrium of $G$ since $F_{2}^{1}(x(\epsilon))=3-2 \epsilon>F_{1}^{1}(x(\epsilon))=\epsilon$ and $F_{2}^{2}(x(\epsilon))=4-$ $2 \epsilon>F_{1}^{2}(x(\epsilon))=1+\epsilon$. Thus, $((1,0),(1,0))$ is a perfect equilibrium since $\quad x(\epsilon)=((\epsilon, 1-\epsilon),(\epsilon, 1-\epsilon))$ $\longrightarrow((1,0),(1,0))$ as $€ \longrightarrow 0$.

\section{Proper Equilibria and Weakly Proper Equilibria}

In 1978, Myerson [11] proposed the notion of proper equilibria for $n$-person finite noncooperative games when he was a visiting scholar in Bielefeld University. This notion requires more rationality for players and seems more reasonable than perfect equilibria. Similar to Myerson's idea, we generalize this concept to population games considering that each agent will try her best to make herself better and better. Furthermore, even if the difference of payoffs of two strategies are sufficiently small, properness also requires the share of the worse one to be sufficiently small, which is too demanding. Agents often have no incentive to change if their choice is not too bad comparing another strategy. Considering this unpleasant requirement of proper equilibria, we introduce the notion of weakly proper equilibria.

We first define the $\epsilon$-proper equilibrium which is stronger than the $\epsilon$-perfect equilibrium.

Definition 8. Let $G=(P, X, F)$ be a population game and let $\epsilon>0$. A completely mixed social state $\bar{x} \in X$ is an $\epsilon$-proper equilibrium of $G$ if it satisfies:

$$
\text { if } F_{i}^{p}(\bar{x})<F_{j}^{p}(\bar{x}) \text {, then } \bar{x}_{i}^{p} \leq \epsilon \bar{x}_{j}^{p}, \quad \forall i, j \in S^{p}, \forall p \in P \text {. }
$$

The basic idea underlying the $\epsilon$-proper equilibrium is that every agent in each population will do her best to 
achieve better strategies although she cannot reach the predictive Nash equilibrium strategy because of the inadequacy of ability. Consequently, the share of a better strategy should be larger than a worse one. Using the concept of $\epsilon$-proper equilibrium, we now introduce the definition of a proper equilibrium.

Definition 9. Let $G=(P, X, F)$ be a population game. A social state $\bar{x} \in X$ is a proper equilibrium of $G$ if there exist some sequences $\left\{\epsilon_{n}\right\}_{n=1}^{\infty}$ and $\left\{\bar{x}\left(\epsilon_{n}\right)\right\}_{n=1}^{\infty}$ satisfying the following:

(1) $\lim _{n \longrightarrow \infty} \epsilon_{n}=0$

(2) $\bar{x}\left(\epsilon_{n}\right)$ is a $\epsilon_{n}$-proper equilibrium of the population game $G$ for each $n$

(3) $\lim _{n \rightarrow \infty} \bar{x}\left(\epsilon_{n}\right)=\bar{x}$

Remark 1. Every proper equilibrium is perfect since each $\epsilon$-proper equilibrium is $\epsilon$-perfect. Then, every proper equilibrium must be a Nash Equilibrium.

The existence of proper equilibria is also easy to establish as long as we can prove the existence of $\epsilon$-proper equilibrium for every sufficiently small $\epsilon$.

Theorem 4. Every population game G possesses at least one proper equilibrium.

Proof. Let $\epsilon \in(0,1)$ and for each sufficiently small $\epsilon$. For any $p \in P$, define vector $\eta^{p}$ by $\left\{\eta^{p_{i}}\right\}\left(\epsilon^{\left|S^{p}\right|} /\left|S^{p}\right|\right)$ for each $i \in S^{p}$, where $|\cdot|$ indicates the cardinality. Then, we have a perturbation of population game $G$. For each $p \in P$, we define a set-valued map $\Phi^{p}: X(\eta)=\prod_{p \in P} X^{p}\left(\eta^{p}\right) \Rightarrow X^{p}\left(\eta^{p}\right)$ by

$$
\Phi^{p}(x)=\left\{y^{p} \in X^{p}\left(\eta^{p}\right): \text { if } F_{i}^{p}(x)<F_{j}^{p}(x) \text {, then } y_{i}^{p} \leq \epsilon y_{j}^{p}, \quad \forall i, j \in S^{p}\right\} .
$$

We first show that, for any $x \in X(\eta), \Phi^{P}(x) \neq \varnothing$. For each $x \in X(\eta)$ and $i \in S^{p}$, we let

$$
\begin{aligned}
g_{i}^{p}(x) & =\left|\left\{k \in S^{p}: F_{i}^{p}(x)<F_{k}^{p}(x)\right\}\right|, \\
y_{i}^{p} & =\frac{\epsilon^{g_{i}^{p}(x)}}{\sum_{k \in S^{p}} \epsilon^{g_{j}^{p}(x)}} .
\end{aligned}
$$

Then, it is obvious that $y^{p} \in \Phi^{p}(x)$.

We next proceed to the proof that $\Phi^{p}(x)$ is a closed and convex set for any $x \in X(\eta)$. For any $y^{p}, z^{p} \in \Phi^{p}(x)$ and $\lambda \in(0,1)$, if $F_{i}^{p}(x)<F_{j}^{p}(x)$, then $y_{i}^{p} \leq \epsilon y_{j}^{p}$ and $z_{i}^{p} \leq \epsilon z_{j}^{p}$ for all $i, j \in S^{p}$. It follows that $\lambda y_{i}^{p}+(1-\lambda) z_{i}^{p}$ $\leq \epsilon\left(\lambda y_{j}^{p}+(1-\lambda) z_{j}^{p}\right)$, which means that $\Phi^{p}(x)$ is a convex set. Assume that $y^{(n) p} \longrightarrow y^{p}$ as $n \longrightarrow \infty$, and if $F_{i}^{p}(x)<F_{j}^{p}(x)$, then $y_{i}^{(n) p} \leq \epsilon y_{j}^{(n) p}$ for all $i, j \in S^{p}$. Passing to the limit, we have $y_{i}^{p} \leq € y_{j}^{p}$, which means that $y^{p} \in \Phi^{p}(x)$, namely, $\Phi(x)$ is a closed set for each $x \in X(\eta)$.

Finally, we prove that the correspondence $\Phi^{p}$ is upper semicontinuous. It suffices to show that the graph of $\Phi^{p}$ is closed. Suppose that $x^{(n)} \longrightarrow x, y^{(n) p} \in \Phi^{p}\left(x^{(n)}\right)$, and $y^{(n) p} \longrightarrow y^{p}$ as $n \longrightarrow \infty$, we show that $y^{p} \in \Phi^{p}(x)$. If $F_{i}^{p}(x)<F_{j}^{p}(x)$, then $F_{i}^{p}\left(x^{(n)}\right)<F_{j}^{p}\left(x^{(n)}\right)$ for sufficiently large $n$ since $x^{(n)} \longrightarrow x$ and payoff function $F$ is continuous. Furthermore, we have $y_{i}^{(n) p} \leq \epsilon y_{j}^{(n) p}$ because $y^{(n) p} \in \Phi^{p}\left(x^{(n)}\right)$. Since $y^{(n) p} \longrightarrow y^{p}$, this implies $y_{i}^{p} \leq \epsilon y_{j}^{p}$ for sufficiently large $n$. Then, we have $y^{p} \in \Phi^{p}(x)$. Therefore, the correspondence $\Phi$ is upper semicontinuous.

For every $x \in X(\eta)$, define $\Phi(x)=\prod_{p \in P} \Phi^{p}(x)$. It is clear that $\Phi$ satisfies all conditions of Lemma 3. So there exists some $x \in X(\eta)$ such that $x \in \Phi(x)$. Obviously, this $x$ is an $\epsilon$-proper equilibrium.

Consequently, from the proof above, for any sequence $\left\{\epsilon_{n}\right\}$ converging to 0 , we have a corresponding sequence $\left\{\bar{x}^{(n)}\right\}$ in which every social state $\bar{x}^{(n)} \in X$ is an $\epsilon_{n}$-proper equilibrium. Furthermore, since $X$ is compact, there exists a convergent subsequence of $\left\{\bar{x}^{(n)}\right\}$ and the limit $\bar{x}$ is clearly a proper equilibrium. The proof is complete.

The following example shows that a perfect equilibrium for population game need not be proper which means the set of proper equilibria may be a proper subset of the set of perfect equilibria.

Example 2. Let $G$ be a population game played by a single population with three strategies. For ease of notation, we omit the superscript for single population games. Thus, the social state space of $G$ is $X=\left\{x \in R_{+}^{3}: x_{1}+x_{2}+x_{3}=1\right\}$. The payoff of $G$ is defined by $F(x)=\left(2 x_{1}+x_{2}, x_{1}+x_{2}+\right.$ $\left.x_{3},-x_{1}-x_{2}-x_{3}\right)$. Obviously, $G$ has two Nash equilibria $(0,1,0)$ and $(1,0,0)$ and both of them are perfect. But only $(1,0,0)$ is proper. Suppose $\epsilon \epsilon(0,1)$ and let $x(\epsilon) \epsilon \operatorname{int} X$ be an $\epsilon$-proper equilibrium of $G$. Because $F_{2}(x(\epsilon))=1>F_{3}(x(\epsilon))=-1 \quad$ and $\quad F_{1}(x \quad(\epsilon))=2 x_{1}$ $+x_{2}>F_{3}(x(\epsilon))=-1$, we have $x_{3}(\epsilon) \leq \epsilon x_{2}(\epsilon)$ and $x_{3}(\epsilon) \leq \epsilon x_{1}(\epsilon)<x_{1}(\epsilon)$. This in turn implies $F_{1}(x(\epsilon))=2 x_{1}(\epsilon)+x_{2}(\epsilon)=1+x_{1}(\epsilon)-x_{3}(\epsilon)>F_{2}(x(\epsilon))$ $=1$ and so $x_{2}(\epsilon) \leq \epsilon x_{1}(\epsilon)$. Since $x_{1}(\epsilon)+x_{2}(\epsilon)+x_{3}(\epsilon)=1$, $x_{1}(\epsilon) \geq 1-\epsilon-\epsilon^{2}$. As $\epsilon \longrightarrow 0$, this $\epsilon$-proper equilibrium converges to $(1,0,0)$. Therefore $(1,0,0)$ is the only proper equilibrium of $G$.

The properness concept requires every agent will try her best to be better, and the shares of better strategies consequently will be larger than that of worse ones. Intuitively, this requirement is undesirable since many agents have no incentives to change if payoffs of two strategies are almost the same. Considering this unreasonable requirement, we introduce the concept of weakly proper equilibria, which requires only that the share of a considerably worse strategy will be smaller. This idea is similar to van Damme [17].

Definition 10. Let $G=(P, X, F)$ be a population game and let $\epsilon>0$. A completely mixed social state $\hat{x} \in X$ is an $\epsilon$-weakly proper equilibrium against some $\bar{x} \in X$ of $G$ if it satisfies:

$$
\text { if } F_{i}^{p}(\bar{x})<F_{j}^{p}(\bar{x}) \text {, then } \widehat{x}_{i}^{p} \leq \epsilon \widehat{x}_{j}^{p}, \quad \forall i, j \in S^{p}, \forall p \in P .
$$

Definition 11. Let $G=(P, X, F)$ be a population game. A social state $\bar{x} \in X$ is a weakly proper equilibrium of $G$ if there 
exist some sequences $\left\{\epsilon_{n}\right\}_{n=1}^{\infty}$ and $\left\{\widehat{x}\left(\epsilon_{n}\right)\right\}_{n=1}^{\infty}$ satisfying the following:

(1) $\lim _{n \longrightarrow \infty} \epsilon_{n}=0$

(2) $\hat{x}\left(\epsilon_{n}\right)$ is a $\epsilon_{n}$-weakly proper equilibrium against $\bar{x}$ of the population game $G$ for each $n$

(3) $\hat{x}\left(\epsilon_{n}\right)$ is an $\epsilon_{n}$-perfect equilibrium of $G$ for each $n$

(4) $\lim _{n \rightarrow \infty} \widehat{x}\left(\epsilon_{n}\right)=\bar{x}$

Remark 2. It is clear from Theorem 3 that every weakly proper equilibrium is perfect since condition (3) in Definition 11 holds.

Next, we give an example which indicates that a weakly proper equilibrium needs not to be proper.

Example 3. Let $G$ be a population game consisting of two populations, and each population has three strategies. Therefore, the social state space is $Z=X \times Y$, where $X=$ $\left\{x \in R_{+}^{3}: x_{1}+x_{2}+x_{3}=1\right\} \quad$ and $\quad Y=\left\{y \in R_{+}^{3}: y_{1}\right.$ $\left.+y_{2}+y_{3}=1\right\}$. Payoffs of $G$ are defined, respectively, by $F^{1}(z)=\left(2 y_{1}+y_{2}+y_{3}, 2 y_{1}+3 y_{3}, 0\right)^{T}$ and $F^{2}(z)=\left(2,1, x_{1}\right.$ $\left.+x_{2}\right)^{T}$ for any $z \in Z$. It is easy to check that the two pure social states $z_{1}=((1,0,0),(1,0,0))$ and $z_{2}=((0,1,0)$, $(1,0,0))$ are the only Nash equilibria of $G$. Similar to the argument in Example 2, we know $z_{1}$ is the only proper equilibrium of $G$. We next show $z_{2}$ is also weakly proper. For $\epsilon \in(0,(1 / 2))$, define a completely mixed social state by $z(\epsilon)=\left(\left(\epsilon, 1-\epsilon-\epsilon^{2}, \epsilon^{2}\right),(1-2 \epsilon, \epsilon, \epsilon)\right)$. From Definitions 7 and 10 , we can easily show that $z(\epsilon)$ is $\epsilon$-perfect and $\epsilon$-weakly proper against $z_{2}$. As $\epsilon$ approaches to $0, z(\epsilon)$ converges to $z_{2}$. Thus, $x(\epsilon)$ and $z_{2}$ satisfy all conditions of Definition 11, which in fact means $z_{2}$ is a weakly proper equilibrium.

We can easily have the existence theorem of weakly proper equilibrium since every proper equilibrium is weakly proper.

Theorem 5. Every population game $G$ possesses at least one weakly proper equilibrium.

Proof. According to Theorem 4, we assume that $\bar{x}$ is a proper equilibrium of $G$. Since each proper equilibrium is perfect, from Theorem 3, there exist sequences $\left\{\epsilon_{n}\right\}_{n=1}^{\infty}$ and $\left\{\hat{x}\left(\epsilon_{n}\right)\right\}_{n=1}^{\infty}$ such that $\lim _{n \longrightarrow \infty} \epsilon_{n}=0, \lim _{n \longrightarrow \infty} \widehat{x}\left(\epsilon_{n}\right)=\bar{x}$, $\widehat{x}\left(\epsilon_{n}\right)$ is an $\epsilon_{n}$-proper equilibrium of the population game $G$ for each $n$, and $\bar{x}$ is a best response against $\hat{x}\left(\epsilon_{n}\right)$ for each $n$. We only need to show that every $\widehat{x}\left(\epsilon_{n}\right)$ is actually $\epsilon_{n}$-weakly proper. If $F_{i}^{p}(\bar{x})<F_{j}^{p}(\bar{x})$, we have $F_{i}^{p}\left(\widehat{x}\left(\epsilon_{n}\right)\right)<F_{j}^{p}\left(\widehat{x}\left(\epsilon_{n}\right)\right)$ for sufficiently large $n$ since $\hat{x}\left(\epsilon_{n}\right) \longrightarrow \bar{x}$ and $F$ is continuous. Furthermore, each $\hat{x}\left(\epsilon_{n}\right)$ is $\epsilon_{n}$-proper equilibrium implying $\widehat{x}_{i}^{p}\left(\epsilon_{n}\right) \leq \epsilon_{n} \widehat{x}_{j}^{p}\left(\epsilon_{n}\right)$, which means every $\widehat{x}\left(\epsilon_{n}\right)$ is $\epsilon_{n}$-weakly proper. The proof is complete.

\section{Robust Equilibria}

In this section, we introduce the concept of robust equilibrium for population games which require only that the share of a considerably worse strategy should be smaller.
Obviously, the idea underlying this concept is similar to weakly proper equilibria. Comparing with weakly proper equilibria, we think our robust equilibria can more intuitively reflect the rationality of each agent. In our robust equilibria, we consider the relative difference of the payoffs of any two strategies.

Definition 12. Let $G=(P, X, F)$ be a population game and let $\epsilon>0$. A completely mixed social state $\bar{x} \in X$ is an $\epsilon$-robust equilibrium of $G$ if it satisfies:

$$
\text { if } F_{i}^{p}(\bar{x})<F_{j}^{p}(\bar{x}) \text {, then } \bar{x}_{i}^{p} \leq \frac{\epsilon}{\widetilde{F}_{j}^{p}(\bar{x})-\widetilde{F}_{i}^{p}(\bar{x})} \bar{x}_{j}^{p}, \quad \forall i, j \in S^{p}, \forall p \in P,
$$

where $\quad \widetilde{F}_{j}^{p}(\bar{x})-\widetilde{F}_{i}^{p}(\bar{x})=\left(F_{j}^{p}(\bar{x}) / \max _{k \in S^{p}} \quad F_{k}^{p}(\bar{x})\right)-$ $\left(F_{i}^{p}(\bar{x}) / \max _{k \in S^{p}} F_{k}^{p}(\bar{x})\right)$ representing the relative payoff difference of the strategies $i$ and $j$.

From the definition of $\epsilon$-robust equilibria, we consider the relative difference of the payoffs of any two strategies. If the difference is sufficiently small, then it is not necessary to require the share of the worse strategy to be smaller. Obviously, this requirement is weaker than that of proper equilibria.

Definition 13. Let $G=(P, X, F)$ be a population game. A social state $\bar{x} \in X$ is a robust equilibrium (our robust equilibrium here is different from van der Laan [18]) of $G$ if there exist some sequences $\left\{\epsilon_{n}\right\}_{n=1}^{\infty}$ and $\left\{\bar{x} \epsilon_{n}\right\}_{n=1}^{\infty}$ satisfying the following:

(1) $\lim _{n \longrightarrow \infty} \epsilon_{n}=0$

(2) $\bar{x}\left(\epsilon_{n}\right)$ is an $\epsilon_{n}$-robust equilibrium of $G$ for each $n$

(3) $\bar{x}\left(\epsilon_{n}\right)$ is an $\epsilon_{n}$-perfect equilibrium of $G$ for each $n$

(4) $\lim _{n \longrightarrow \infty} \bar{x}\left(\epsilon_{n}\right)=\bar{x}$

Obviously, any interior Nash equilibrium $\bar{x}$ is robust since each component of $F(x)$ is the same. Due to condition (3) of Definition 13, each robust equilibrium is naturally an Nash equilibrium.

Lemma 6. Let $G=(P, X, F)$ be a population game. Then, every robust equilibrium of $G$ is a Nash equilibrium of $G$.

We notice that each proper equilibrium is robust, and then, the existence of robust equilibrium is obtained.

Theorem 6. Every population game G possesses at least one robust equilibrium.

Proof. Assume that $\bar{x}$ is a proper equilibrium of $G$. We next show $\bar{x}$ must be a robust equilibrium of $G$. From Theorem 3, there exist sequences $\left\{\epsilon_{n}\right\}_{n=1}^{\infty}$ and $\left\{\hat{x}\left(\epsilon_{n}\right)\right\}_{n=1}^{\infty}$ such that $\lim _{n \longrightarrow \infty} \epsilon_{n}=0, \lim _{n \longrightarrow \infty} \widehat{x}\left(\epsilon_{n}\right)=\bar{x}, \widehat{x}\left(\epsilon_{n}\right)$ is an $\epsilon_{n}$-proper equilibrium of the population game $G$ for each $n$, and $\bar{x}$ is a best response against $\widehat{x}\left(\epsilon_{n}\right)$ for each $n$. Since $\hat{x}\left(\epsilon_{n}\right)$ is an $\epsilon_{n}$-proper equilibrium of $G$, we have

$$
\text { if } F_{i}^{p}\left(\widehat{x}\left(\epsilon_{n}\right)\right)<F_{j}^{p}\left(\widehat{x}\left(\epsilon_{n}\right)\right) \text {, then } \widehat{x}_{i}^{p}\left(\epsilon_{n}\right) \leq \epsilon_{n} \widehat{x}_{j}^{p}\left(\epsilon_{n}\right), \quad \forall i, j \in S^{p}, \forall p \in P .
$$


On the one hand, suppose that $F_{j}^{p}\left(\widehat{x}\left(\epsilon_{n}\right)\right)-F_{i}^{p}\left(\hat{x}\left(\epsilon_{n}\right)\right) \nrightarrow 0$ as $n \longrightarrow \infty$. We can assume without loss of generality that the sequence $\left\{\hat{x}\left(\epsilon_{n}\right)\right\}$ such that $F_{j}^{p}\left(\hat{x}\left(\epsilon_{n}\right)\right)-F_{i}^{p}\left(\hat{x}\left(\epsilon_{n}\right)\right) \longrightarrow 0$ has a positive lower bound as $n$ is large enough. Thus, we have

$$
\widehat{x}_{i}^{p}\left(\epsilon_{n}\right) \leq \frac{\epsilon_{n}}{\widetilde{F}_{j}^{p}\left(\widehat{x}\left(\epsilon_{n}\right)\right)-\widetilde{F}_{i}^{p}\left(\widehat{x}\left(\epsilon_{n}\right)\right)} \widehat{x}_{j}^{p}\left(\epsilon_{n}\right),
$$

where $\quad F_{j}^{p}\left(\widehat{x}\left(\epsilon_{n}\right)\right)-F_{i}^{p}\left(\widehat{x}\left(\epsilon_{n}\right)\right)=\left(F_{j}^{p}\left(\widehat{x}\left(\epsilon_{n}\right)\right) / \max _{k \in S P} F_{k}^{p}\right.$ $\left.\left(\widehat{x}\left(\epsilon_{n}\right)\right)\right)-\left(F_{i}^{p}\left(\widehat{x}\left(\epsilon_{n}\right)\right) / \max _{k \in S^{p}} F_{k}^{p}\left(\hat{x}\left(\epsilon_{n}\right)\right)\right)$.

On the other hand, suppose that $F_{j}^{p}\left(\hat{x}\left(\epsilon_{n}\right)\right)-F_{i}^{p}\left(\hat{x}\left(\epsilon_{n}\right)\right) \longrightarrow 0$ as $n \longrightarrow \infty$, then as long as $n$ is large enough, $\hat{x}_{i}^{p}\left(\epsilon_{n}\right) \leq \epsilon_{n} \hat{x}_{j}^{p}\left(\epsilon_{n}\right)$ also implies (28) holds. The proof is complete.

Since the underlying idea of robust equilibria for population games are the same as that of weakly proper equilibria, we next show that these two concepts are actually equivalent.

Theorem 7. Let $G=(P, X, F)$ be a population game. A social state $\bar{x}$ is a weakly proper equilibrium of $G$ if and only if $\bar{x}$ is a robust equilibrium of $G$.

Proof. Assume that $\bar{x}$ is a weakly proper equilibrium of $G$. Then, there exist $\left\{\epsilon_{n}\right\}_{n=1}^{\infty}$ and $\left\{\bar{x}\left(\epsilon_{n}\right)\right\}_{n=1}^{\infty}$ such that $\lim _{n \longrightarrow \infty} \epsilon_{n}=0, \lim _{n \longrightarrow \infty} \bar{x}\left(\epsilon_{n}\right)=\bar{x}, \bar{x}\left(\epsilon_{n}\right)$ is an $\epsilon_{n}$-weakly proper, and $\epsilon_{n}$ perfect equilibrium of the population game $G$ for each $n$. If $F_{i}^{p}\left(\bar{x}\left(\epsilon_{n}\right)\right)<F_{j}^{p}\left(\bar{x}\left(\epsilon_{n}\right)\right)$, then $F_{i}^{p}(\bar{x})<F_{j}^{p}(\bar{x})$ as $n \longrightarrow \infty$. On the one hand, if $F_{i}^{p}(\bar{x})<F_{j}^{p}(\bar{x})$, we have $\bar{x}_{i}^{p}\left(\epsilon_{n}\right) \leq \epsilon_{n} \bar{x}_{j}^{p}\left(\epsilon_{n}\right)$ for sufficiently large $n$ due to the weak properness of each $\bar{x}\left(\epsilon_{n}\right)$. Furthermore, as long as $n$ is large enough, we have

$$
\bar{x}_{i}^{p}\left(\epsilon_{n}\right) \leq \frac{\epsilon_{n}}{\widetilde{F}_{j}^{p}\left(\bar{x}\left(\epsilon_{n}\right)\right)-\widetilde{F}_{i}^{p}\left(\bar{x}\left(\epsilon_{n}\right)\right)} \bar{x}_{j}^{p}\left(\epsilon_{n}\right),
$$

where $\widetilde{F}_{j}^{p}\left(\bar{x}\left(\epsilon_{n}\right)\right)-\widetilde{F}_{i}^{p}\left(\bar{x}\left(\epsilon_{n}\right)\right)=\left(F_{j}^{p}\left(\bar{x}\left(\epsilon_{n}\right)\right) / \max _{k \in S^{p}}\right.$ $\left.F_{k}^{p}\left(\bar{x}\left(\epsilon_{n}\right)\right)\right)-\left(F_{i}^{p}\left(\bar{x}\left(\epsilon_{n}\right)\right) / \max _{k \in S^{P}} F_{k}^{p}\left(\bar{x}\left(\epsilon_{n}\right)\right)\right) \quad$ since $\epsilon_{n} \longrightarrow 0, \bar{x}\left(\epsilon_{n}\right) \longrightarrow \bar{x}$, and $F_{j}^{p}\left(\bar{x}\left(\epsilon_{n}\right)\right)-F_{i}^{p}\left(\bar{x}\left(\epsilon_{n}\right)\right) \rightarrow 0$. Thus, we can find a subsequence of $\left\{\bar{x}\left(\epsilon_{n}\right)\right\}_{n=1}^{\infty}$ converges to $\bar{x}$ and each element of this subsequence is a $\epsilon_{n}$-robust equilibrium of $G$. On the other hand, if $F_{i}^{p}(\bar{x})<F_{j}^{p}(\bar{x})$, then $F_{i}^{p}\left(\bar{x}\left(\epsilon_{n}\right)\right)<F_{j}^{p}\left(\bar{x}\left(\epsilon_{n}\right)\right)$ as $n$ is large enough. Accordingly, (29) naturally holds from the knowledge of logic.

Conversely, suppose that $\bar{x}$ is a robust equilibrium of $G$. Then, there exist $\left\{\epsilon_{n}\right\}_{n=1}^{\infty}$ and $\left\{\bar{x}\left(\epsilon_{n}\right)\right\}_{n=1}^{\infty}$ such that $\lim _{n \longrightarrow \infty} \epsilon_{n}=0, \lim _{n \longrightarrow \infty} \bar{x}\left(\epsilon_{n}\right)=\bar{x}, \bar{x}\left(\epsilon_{n}\right)$ is an $\epsilon_{n}$-robust proper, and $\epsilon_{n}$-perfect equilibrium of the population game $G$ for each $n$. If $F_{i}^{p}(\bar{x})<F_{j}^{p}(\bar{x})$, then $F_{i}^{p}\left(\bar{x}\left(\epsilon_{n}\right)\right)<F_{j}^{p}\left(\bar{x}\left(\epsilon_{n}\right)\right)$ for sufficiently large $n$. Since each $\bar{x}\left(\epsilon_{n}\right)$ is an $\epsilon_{n}$-robust equilibrium of $G$, we have

$$
\bar{x}_{i}^{p}\left(\epsilon_{n}\right) \leq \frac{\epsilon_{n}}{\widetilde{F}_{j}^{p}\left(\bar{x}\left(\epsilon_{n}\right)\right)-\widetilde{F}_{i}^{p}\left(\bar{x}\left(\epsilon_{n}\right)\right)} \bar{x}_{j}^{p}\left(\epsilon_{n}\right),
$$

where $\quad \widetilde{F}_{j}^{p}\left(\bar{x}\left(\epsilon_{n}\right)\right)-\widetilde{F}_{i}^{p}\left(\bar{x}\left(\epsilon_{n}\right)\right)=\left(F_{j}^{p}\left(\bar{x}\left(\epsilon_{n}\right)\right) / \max _{k \in S^{p}} F_{k}^{p}\right.$ $\left.\left(\bar{x}\left(\epsilon_{n}\right)\right)\right)-\left(F_{i}^{p}\left(\bar{x}\left(\epsilon_{n}\right)\right) / \max _{k \in S^{p}} F_{k}^{p}\left(\bar{x}\left(\epsilon_{n}\right)\right)\right)$.

For each $n$, we set $\epsilon_{n}^{\prime}=\left(\epsilon_{n} / \widetilde{F}_{j}^{p}\left(\bar{x}\left(\epsilon_{n}\right)\right)-\widetilde{F}_{i}^{p}\left(\bar{x}\left(\epsilon_{n}\right)\right)\right)$. Then, $e_{n}^{\prime} \longrightarrow 0$ as $n \longrightarrow \infty$ and (30) implies

$$
\bar{x}_{i}^{p}\left(\epsilon_{n}\right) \leq \epsilon_{n}^{\prime} \bar{x}_{j}^{p}\left(\epsilon_{n}\right)
$$

for sufficiently large $n$. Thus, we obtain a subsequence of $\left\{\bar{x}\left(\epsilon_{n}\right)\right\}_{n=1}^{\infty}$ in which each element is an $\epsilon_{n}^{\prime}$-weakly proper equilibrium of $G$. The proof is complete.

We conclude this section with the following two examples.

Example 4. We return to the population game $G$ of Example 3. From Example 3, we know $z_{1}$ is proper and it is easy to verify that $z_{1}$ is robust. We notice that $z_{2}$ is also robust. Set $z(\epsilon)=\left(\left(\epsilon, 1-\epsilon-\epsilon^{3}, \epsilon^{3}\right),\left(1-2 \epsilon^{2}, \epsilon^{2}, \epsilon^{2}\right)\right)$. We can easily show $z(\epsilon)$ is $\epsilon$-perfect and $\epsilon$-robust as long as $\epsilon$ is small enough.

Example 5. Let $G$ be a population game played by a single population with three strategies. The payoff of $G$ is defined by $F(x)=\left(1+x_{1}-x_{3}, 1,-1\right)$. Obviously, $G$ has two Nash equilibria $(0,1,0)$ and $(1,0,0)$, and both of them are perfect. Similar to Example 3.1, population state $(1,0,0)$ is the only proper and thus robust. We assume that the state $(0,1,0)$ is robust. Since for any $x \in X, F_{1}(x)>F_{3}(x)$, according to the definition of $\epsilon$-robust equilibrium, $x_{3}<x_{1}$. Furthermore, we have $F_{1}(x)>F_{2}(x)$ and $x_{2} \leq\left(\epsilon / \widetilde{F}_{1}(x)>\widetilde{F}_{2}(x)\right) x_{1}$, which means that $x_{2}<x_{1}$. This implies that we can never find a sequence converging to $(0,1,0)$. Therefore, the population state $(0,1,0)$ is not a robust equilibrium.

\section{Conclusion}

This paper has generalized the notions of perfect equilibria, proper equilibria, and weakly proper equilibria to models of population games. And we have proposed the so-called robust equilibrium for population games. Lemma 4 is an interesting and important result for perturbed population games. We have shown the existences of the above equilibria without the assumption of the convexity of the payoff. Furthermore, we have proved that our robust equilibrium is equivalent to the weakly robust equilibrium. From Example 1, we have seen the perfectness really refines the Nash equilibria for population games, and from Example 2, the properness really refines perfect equilibria. Example 3 shows the set of proper equilibria may be a proper subset of weakly proper equilibria. From Example 5 , we have seen the robustness concept is a strict refinement of perfectness concept. For the future work, we will consider the dynamical stability of the mentioned refinement concepts.

\section{Data Availability}

No data were used to support this study.

\section{Disclosure}

Part of this work was done at Tilburg University while the author was a visiting scholar of Econometrics and Operations Research. 


\section{Conflicts of Interest}

The authors declare that they have no conflicts of interest.

\section{Acknowledgments}

The authors would like to thank Professor Dolf Talman for his kind help and valuable comments on the topic of the paper. The authors gratefully acknowledge the supportprovided by Guizhou University Innovation Fund for Graduate Student Project (Grant no. 2014067).

\section{References}

[1] J. Nash, "Non-cooperative games," The Annals of Mathematics, vol. 54, no. 2, pp. 286-295, 1951.

[2] J. F. Nash, Non-cooperative games, Ph.D. thesis, Mathematics Department, Princeton University, Princeton, NJ, USA, 1950.

[3] J. W. Weibull, "The mass action interpretation," Journal of Economic Theory, vol. 69, pp. 165-171, 1995.

[4] H. W. Kuhn, J. C. Harsanyi, R. Selten et al., "The work of John F. Nash Jr. in game theory: Nobel Seminar, 8 December 1994," Duke Mathematical Journal, vol. 81, no. 1, pp. 1-29, 1995.

[5] E. E. C. van Damme and J. W. Weibull, "Equilibrium in strategic interaction: the contributions of John C. Harsanyi, John F. Nash and Reinhard Selten," The Scandinavian Journal of Economics, vol. 97, no. 1, pp. 15-40, 1995.

[6] S. J. Maynard and G. R. Price, "The logic of animal conflict," Nature, vol. 246, pp. 15-18, 1973.

[7] S. J. Maynard, Evolution and the Theory of Games, Cambridge Univrsity Press, Cambridge, UK, 1982.

[8] J. W. Weibull, Evolutionary Game Theory, MIT Press, Cambridge, MA, USA, 1995.

[9] W. H. Sandholm, Population Games and Evolutionary Dynamics, MIT Press, Cambridge, MA, USA, 2010.

[10] E. Kalai and D. Samet, "Persistent equilibria in strategic games," International Journal of Game Theory, vol. 13, no. 3, pp. 129-144, 1984.

[11] R. B. Myerson, "Refinements of the Nash equilibrium concept," International Journal of Game Theory, vol. 7, no. 2, pp. 73-80, 1978.

[12] A. Okada, "On stability of perfect equilibrium points," International Journal of Game Theory, vol. 10, no. 2, pp. 67-73, 1980.

[13] R. Selten, "Reexamination of the perfectness concept for equilibrium points in extensive games," International Journal of Game Theory, vol. 4, no. 1, pp. 25-55, 1975.

[14] W. T. Wu and J. H. Jiang, "Essential equilibrium points of n-person noncooperative games," Scientia Sinica, vol. 11, pp. 1307-1322, 1962.

[15] J. S. Pang, Finite Dimensional Variational Inequalities and Complementarity Problems, Vol. 1, Springer, Berlin, Germany, 2003.

[16] J. P. Aubin and I. Ekeland, Applied Nonlinear Analysis, Wiley, New York, NY, USA, 1984.

[17] E. E. C. van Damme, Stability and Perfection of Nash Equilibria, Springer-Verlag, Berlin, Germany, 2nd edition, 1991.

[18] G. van der Laan, A. J. J. Talman, and Z. Yang, "Refinements of stationary points with applications to noncooperative games and economics," SIAM Journal on Optimization, vol. 16, no. 3, pp. 854-870, 2006. 